\title{
Uso y presencia de las tecnologías en personas con discapacidad
}

\author{
Hernández Sánchez, Brizeida \\ Universidad Especializada de las Américas \\ Panamá, Panamá \\ ORCID 0000-0001-5397-1546 \\ González, Cedeño Greisy \\ Instituto Panameño de Habilitación Especial \\ Panamá, Panamá \\ ORCID 0000-0003-3464-8019
}

\begin{abstract}
The moment is crucial to respond to an invisible population that urgently requires opportunities to employ themselves, have a life plan and contribute to their community. People with disabilities need to overcome barriers that hinder the use of technology, working from their attitudes. On the other hand, the World Report on Disability of the World Health Organization (2011) found significant disadvantages at the educational level and, therefore, in the labor market, with a labor rate of $44 \%$ compared to people without disabilities in the United States. $75 \%$. Therefore, autonomy and independence are basic rights. The research was based on the quality-of-life model of Schalock and Verdugo (2002). The objective of the research identified the presence and usefulness of digital tools in people with disabilities. The research focused on knowing what tools would give them advantages, what tools they need to work; in addition, training needs were identified. These objectives led us to make assumptions: To what extent digital skills affect people with disabilities. The research methodology involved the participation of 257 people with disabilities, in nine provinces. $51.7 \%$ male, 48 . $2 \%$ female. A survey-type instrument was developed for people with disabilities. The instrument was drawn up from the following sources: a) review of the scientific literature on disability and technology and its impact on job placement and programs; b) report of associations that work for, with and for people with intellectual disabilities and c) repositories that collect information on activities of people with disabilities. The technique was by phone calls using the WhatsApp application applied between December 18, 2020, to February 28, 2021. Among the aspects evaluated are technology for daily use, devices and usability or challenges of technology for people
\end{abstract}


with disabilities. The first results express that $57.5 \%$ have had some work experience and $42.4 \%$ have no relationship with employment. The variety of situations collected allows us to design a complete map in relation to whether the technologies help and impact the quality of life.

Keywords: technologies, disability, equity, associations, quality of life.

\section{Resumen}

El momento es crucial para responder a una población invisible que requiere de forma impostergable oportunidades para emplearse, tener un plan de vida y aportar a su comunidad. Las personas con discapacidad requieren superar barreras que obstaculizan la utilización de la tecnología trabajando desde las actitudes. Por otro lado, el Informe Mundial sobre la Discapacidad de la Organización Mundial de la Salud (2011) comprobó desventajas significativas a nivel educativo $y$, por ende, en el mercado laboral, con una tasa laboral del $44 \%$ frente a las personas sin discapacidad del $75 \%$. Por lo anterior, la autonomía y la independencia son derechos básicos. La investigación se basó en el modelo de calidad de vida de Schalock y Verdugo (2002) [1]. El objetivo de la investigación identificó la presencia y utilidad de las herramientas digitales en las personas con discapacidad. La investigación se enfocó en conocer qué herramientas les darían ventajas, qué herramientas necesitan para trabajar; además, se identificó las necesidades de formación. Estos objetivos nos llevaron a planearnos supuestos: En qué medida competencias digitales inciden en las personas con discapacidad. La metodología de la investigación implicó la participación de 257 personas con discapacidad, en nueve provincias. 51,7\% masculinos, 48 . 2\% femenino. Se elaboró un instrumento tipo encuesta dirigido a personas con discapacidad. La elaboración del instrumento se realizó a partir de las siguientes fuentes: a) revisión de la literatura científica sobre discapacidad y tecnología y su impacto en la inserción laboral y los programas; b) informe de las asociaciones que trabajan para, con y por las personas con discapacidad intelectual y c) repositorios que recopilan información sobre actividades de las personas con discapacidad. La técnica fue por llamadas de teléfono usando la aplicación WhatsApp aplicada entre el 18 de diciembre 2020 a 28 febrero 2021. Entre los aspectos evaluados están: tecnología de uso diario; dispositivos y usabilidad o retos de la tecnología para las personas con discapacidad. Los primeros resultados expresan que $57,5 \%$ han tenido alguna experiencia laboral y $42,4 \%$ no tiene ninguna relación con el empleo. La variedad de situaciones recogidas nos permite diseñar un mapa completo en relación, si las tecnologías ayudan e impactan en la calidad de vida.

Palabras claves: tecnologías, discapacidad, equidad, asociaciones, calidad de vida. 


\section{INTRODUCCIÓN}

La inclusión en el mercado laboral es un proceso complejo para todos, especialmente para las personas con discapacidad, debido a los prejuicios que crean numerosas barreras. El empleo inclusivo es la actividad laboral dirigida y adaptada a personas que padecen algún tipo de discapacidad, intelectual, física o emocional. Es necesario aclarar los conceptos de "empleo inclusivo " $y$ " empresa inclusiva. "El primero se refiere a la implementación de medidas multidisciplinares enfocadas a mejorar la empleabilidad de un grupo de personas con peculiaridades, que les impiden competir en el mercado laboral con las mismas ventajas que el resto. El segundo concepto se utiliza para referirse a los negocios que implican un grupo con dificultades financieras, pero que no necesariamente sufren alguna limitación.

Los avances tecnológicos han permitido abrir tres caminos imprescindibles para la inclusión: 1) la oferta de cursos presenciales o formaciones a distancia dirigidas a mejorar la cualificación de este colectivo en la gestión o gestión empresarial; 2) la introducción y popularización del teletrabajo, algo que ha ganado protagonismo durante la pandemia; y 3) la búsqueda de empleo específicamente dirigido a personas con discapacidad. Y es precisamente en este tercer punto donde se centran las propuestas para promover el empleo inclusivo.

La inclusión de las personas con discapacidad en el mercado laboral se entiende como un factor decisivo para la inclusión social, la independencia económica y la consecuente valorización y realización de estos ciudadanos. Las personas con discapacidad se mencionan en varias partes de los Objetivos de Desarrollo Sostenible (ONU, 2015) [2] y, específicamente, en las partes relacionadas con la educación, el crecimiento y el empleo, la desigualdad, la accesibilidad de los asentamientos humanos. De los 17 objetivos de la Agenda 2030, se destacan los siguientes relacionados con la discapacidad: Objetivo 4: Educación inclusiva y equitativa de calidad y promoción de oportunidades de aprendizaje a lo largo de la vida para todos. Además, la propuesta pide la construcción y mejora de instalaciones educativas que sean sensibles a los niños, las discapacidades y el género; Objetivo 8 : promover el crecimiento económico sostenido, inclusivo y sostenible, el empleo pleno y productivo y el trabajo decente para todos, incluidas las personas con discapacidad; Objetivo 10 , que se esfuerza por reducir la desigualdad dentro y entre los países mediante el empoderamiento y la promoción de la inclusión social, económica y política de todos, incluidas las personas con discapacidad ; Objetivo 11: proporcionar acceso a sistemas de transporte seguros, asequibles, accesibles y sostenibles, mejorando la seguridad vial, en particular mediante la expansión del transporte público, con especial atención a las necesidades de las personas en situaciones vulnerables, como las personas con discapacidad; y el Objetivo 17: aumento de la calidad y la puntualidad de los datos desglosados por discapacidad .

La velocidad de desarrollo de la sociedad de la información actual se ve como una desventaja para las personas con discapacidad, ya que requiere una mayor eficiencia en 
el tratamiento e interpretación de la información. Las tecnologías de la información y la comunicación (TIC) son hoy en día la ruta que seguir para la transformación social y económica. El uso de la tecnología en personas con discapacidad puede considerarse otro aspecto del ajuste a sus necesidades (Tanis et al., 2012) [3]. En los últimos años, las TIC se han utilizado como una herramienta para mejorar la calidad de vida de las personas de una forma más eficiente y eficaz. Existen varias herramientas que ayudan a las personas con discapacidad, brindándoles oportunidades de aprendizaje, aumentando su potencial en diferentes ámbitos de sus vidas, al difuminar las desigualdades (Mishra et al., 2010) [4]. Estas tecnologías dan mayor libertad a las personas con discapacidad y a sus familias, ya que las personas con discapacidad pueden ser más independientes, aumentando su calidad de vida (Lorah et al., 2015) [5]. Las TIC son una gran esperanza para las personas con discapacidad: pueden reducir las barreras que pueden perjudicar o impedir que las personas con discapacidad participen en las actividades cotidianas (D’Aubin, 2007) [6]. Las tecnologías han adquirido un significado especial en la mejora y normalización de la vida de las personas con discapacidad, así como en su acceso al empleo, permitiéndoles desarrollarse, tanto a nivel personal como laboral. El estudio identificó la presencia y utilidad de las herramientas digitales en las personas con discapacidad, se buscó conocer qué herramientas les darían ventajas, qué herramientas necesitan para trabajar; además, se identificó las necesidades de formación. Estos objetivos nos llevaron a planearnos supuestos: En qué medida competencias digitales inciden en las personas con discapacidad.

\section{MÉTODO}

La metodología de la investigación es cuantitativa con un diseño cuasiexperimento, han participado personas con discapacidad, nueve provincias. $51,7 \%$ masculinos, 48 . $2 \%$ femenino. Se elaboró un instrumento tipo encuesta dirigido a personas con discapacidad. La elaboración del instrumento se realizó a partir de las siguientes fuentes: a) revisión de la literatura científica sobre discapacidad y tecnología y su impacto en la inserción laboral y los programas; $y$ b) informe de las asociaciones que trabajan para, con y por las personas con discapacidad intelectual.

La técnica de recolección fue por llamadas de teléfono usando la aplicación WhatsApp aplicada entre el 18 de diciembre 2020 a 28 febrero 2021. El objetivo principal del estudio fue valorar cómo indicen las herramientas digitales en la persona con discapacidad. Por esta razón, el instrumento se enfocó en recoger datos en las siguientes categorías: Preguntas generales, Tecnología de uso diario, Dispositivos y usabilidad. Retos de la tecnología para las personas con discapacidad. Obstáculos de la tecnología para las personas con discapacidad. Herramientas digitales para el trabajo. Programas de formación existentes. 
El cuestionario tiene un lenguaje pertinente y apropiado que permita la comprensión de los participantes. La población de estudio estará conformada por personas con discapacidad de la Federación Nacional Amigos de personas con discapacidad de la República de Panamá. Para la selección de la muestra se realizó un muestreo de tipo probabilístico. Este tipo de estudio mantiene, semejanzas con el muestreo aleatorio estratificado, pero no mantiene el carácter de aleatoriedad. Se extraerá una muestra también base a las variables de, sexo, edad, contexto, experiencias previas.

\section{RESULTADOS}

Los resultados se recogen en 6 bloques: (1) Aspecto laboral y acceso a tecnología; (2) Tecnologías de uso diario; (3) Usabilidad; (4) Retos de tecnología; (5) Obstáculos de la tecnología; y (6) Herramientas digitales. El tipo de respuesta varía según la pregunta o bloques: hay respuestas dicotómicas de "si / no"; de opciones (simples o múltiples); y respuestas de escala Likert de 5 puntos.

Aspecto laboral y acceso a tecnología en este sub-bloque, encontramos $57.5 \%$ de los participantes en la encuesta tiene experiencia laboral, $24.4 \%$ se encuentra trabajando en la actualidad. Además, se les ha preguntado que si creen que las tecnologías pueden ayudar a mejorar el empleo y más del $90 \%$ han respondido que sí les ayudarían.

En cuanto a la situación laboral, vemos que hay casi el triple de contratos a tiempo completo que a tiempo parcial $(19.2 \%$ vs. $6.8 \%)$ y que solo el $7.5 \%$ trabaja de forma independiente. Hay un $17.7 \%$ que no dedica su tiempo ni a trabajar ni a estudiar, y un 20.7\% que se encuentra estudiando actualmente.

En la usabilidad, el uso de internet de los encuestados se les ha respondido: (1) uso habitual de internet, (2) la frecuencia de acceso y (3) las formas de conectarse.

- Uso habitual de internet. El general sus medias oscilan entre valores medios (2.75 y 3.91). Cabe destacar que la concentración más alta de puntos se encuentra en el valor 5 , siendo este el valor más alto de la escala Likert.

- Frecuencia de acceso. Casi el $50 \%$ de los encuestados han respondido con el valor más alto de esta escala (5), lo que significa que la gran mayoría accede a internet con bastante frecuencia.

- Formas de conectarse. En este bloque se encuentra una diferencia importante ya que la mitad de las personas del estudio acceden a internet mediante zona wifi de manera habitual. 
Tabla 1: Análisis descriptivo. Uso de internet. $\mathrm{N}=266$ participantes

\begin{tabular}{|c|c|c|c|c|c|c|}
\hline \multirow[b]{2}{*}{ ÍTEM } & \multicolumn{5}{|c|}{$\%$ de respuesta de cada opción } & \multirow{2}{*}{ Media } \\
\hline & 1 & 2 & 3 & 4 & 5 & \\
\hline $\begin{array}{l}\text { USO DE INTERNET } \\
\text { tengo formación }\end{array}$ & 10.9 & 13.5 & 21.1 & 19.9 & 34.6 & 3.54 \\
\hline aprender experimentando & 6.0 & 9.8 & 16.2 & 23.7 & 44.4 & 3.91 \\
\hline cursos sobre tecnología & 34.6 & 15.4 & 13.9 & 12.4 & 23.7 & 2.75 \\
\hline $\begin{array}{l}\text { FRECUENCIA DE ACCESO } \\
\text { varias veces al día }\end{array}$ & 11.3 & 7.9 & 16.2 & 21.1 & 43.6 & 3.78 \\
\hline Todos los días & 9.8 & 8.3 & 18.0 & 18.8 & 45.1 & 3.81 \\
\hline Varias veces a la semana & 12.4 & 6.4 & 15.8 & 19.9 & 45.5 & 3.80 \\
\hline $\begin{array}{l}\text { FORMAS DE CONECTARSE } \\
\text { Zonas wifi }\end{array}$ & 26.7 & 3.0 & 6.8 & 12.8 & 50.8 & 3.58 \\
\hline Prepago & 38.0 & 17.3 & 15.8 & 7.1 & 21.8 & 2.58 \\
\hline Contrato residencial & 46.2 & 3.0 & 4.9 & 7.9 & 38.0 & 2.88 \\
\hline
\end{tabular}

Uso y utilidad de herramientas digitales, en este bloque hemos encontrado la media más alta en la pregunta de si usan mensajería instantánea (3.76) puesto que la mayoría de las puntuaciones se encuentran entre los valores altos (4 y 5) y que el $42.1 \%$ de los encuestados respondió con el valor más alto de la escala Likert. El valor medio más bajo (1.77) pertenece a si compran online donde el $71.4 \%$ respondió con Likert más baja (1); a éste le sigue el uso para leer noticias (2.84). El resto, se encuentran entre los valores promedio: 3.30 y 3.70 puntos.

Tenencia de dispositivos los participantes del estudio, se les preguntó sobre si tienen o no tienen los dispositivos televisión, radio, computadora, tableta, teléfono celular, contrato de internet, internet público, redes sociales. El dispositivo que más abunda entre estas personas es el celular propio (casi un 90\%), mientras que por el contrario solo un $3 \%$ dispone de un iPod. Se puede destacar respecto a los resultados obtenidos que hay más personas que tienen un laptop (35.0\%) en vez de una computadora fija (26.3\%).

Retos de la tecnología, búsqueda de información a través de internet. Los ítems de este tipo Likert. Según las medias de los resultados obtenidos la mayoría de las personas que participan en la encuesta utiliza el internet como fuente principal para buscar información ya que su valor medio es el más elevado (3.64) que se debe a que se concentra la mayor parte de las respuestas en el valor más alto de la escala Likert (39.1\%).

Acceso a la tecnología a personas con discapacidad intelectual. A la vista de los valores medios obtenidos, se comprueba que el valor más alto se encuentra en el acceso a internet por recursos propios (3.68) con casi un 50\% que ha respondido con el máximo valor Likert, lo que nos lleva a concluir que la mayoría de las personas disponen de acceso por este medio. Se puede destacar que la siguiente media más alta es el no contar con tecnología (2.05) con un elevado número de casos (56.0\%) que ha emitido la respuesta más baja de la escala; en tanto que el resto de los reactivos tienen valores promedio inferiores a los 2 puntos. 


\section{CONCLUSIONES}

Luego de realizada la investigación se retoman los objetivos planificados, el análisis realizado nos muestra la existencia de una relación significativa $(p<.001)$ con un grado de intensidad moderado (.26); pero suficiente como para poder concluir que las variables están relacionadas entre sí. La relación se define por la asociación de los valores altos entre sí, lo que nos lleva a la conclusión de que tienden a estar más satisfechos aquellos que están más en acuerdo con la afirmación de que las tecnologías son un factor de exclusión. No obstante, también se aprecia una cierta presencia de casos cuyas respuestas son asocian al contario: más satisfacción personal estando menos de acuerdo con la afirmación de exclusión.

\section{Referencias}

[1] Schalock, R. L., Verdugo, M. A., \& Braddock, D. L. (2002). Handbook on quality of life for human service practitioners. Washington, DC: American Association on Mental Retardation.

[2] ONU. (2015). Objetivos de Desarrollo Sostenible Programa de las Naciones Unidas para el Desarrollo

[3] Lorah , ER, Parnell, A., Whitby, PS y Hantula , D. (2015). Una revisión sistemática de las tabletas y los reproductores multimedia portátiles como dispositivos generadores de voz para personas con trastorno del espectro autista. Revista de autismo y trastornos del desarrollo, 45 (12), 37923804. https://doi.org/10.1007/s10803-014-2314-4

[4] Michailakis, D. (2001). Las tecnologías de la información y la comunicación y las oportunidades de las personas discapacitadas en el mercado laboral sueco , 16 (4), 477-500. DOI: 10.1080 / 09687590120059496

[5] Mishra, M. Sharma, V. y Tripathi, R. (2010). Las TIC como herramienta de enseñanza y aprendizaje en relación con el alumno con discapacidad. Commonwealth of Learning - Aprendizaje para el desarrollo sostenible.

[6] A. D'Aubin. Working for barrier removal in the ICT area: creating a more accessible and inclusive Canada: a position statement by the Council of Canadians with disabilities. The Information Society, 23(3), 193-201.2007.

[7] Tanis, S., Palmer, S., Wehmeyer, M., Davies, K., Stock, E., Lobb, K. y Bishop, B. (2012). Encuesta de autoinforme basada en computadora sobre el uso de tecnología por personas con discapacidades intelectuales y del desarrollo. Discapacidades intelectuales y del desarrollo, 50, 53-68. doi: 10.1352 / 1934-9556-50.1.53

[8] UNESCO. (2009). La UNESCO y Sun Microsystems anuncian un esfuerzo conjunto de educación y desarrollo comunitario impulsado por tecnologías abiertas. Comunicado de prensa de la UNESCO.

\section{Autorización y Licencia CC}

Los autores autorizan a APANAC XVIII a publicar el artículo en las actas de la conferencia en Acceso Abierto (Open Access) en diversos formatos digitales (PDF, HTML, EPUB) e integrarlos en diversas plataformas online como repositorios y bases de datos bajo la licencia, Attribution-NonCommercialShareAlike 4.0 International (CC BY-NC-SA 4.0) https://creativecommons.org/licenses/by-nc-sa/4.0/. Ni APANAC XVIII ni los editores son responsables ni del contenido ni de las implicaciones de lo expresado en el artículo. 